\title{
Comparative Study of Response Surface Methodology and Artificial Neural Network for Modeling and Optimization of Extraction Process Parameters on Tetrapleura Tetraptera
}

\author{
*OYEDOH, EA; ERUMI, GA; AKHABUE, CE \\ Department of Chemical Engineering, Faculty of Engineering, University of Benin, Benin City, Nigeria \\ *Corresponding Author Email: egheoyedoh@uniben.edu
}

\begin{abstract}
Bioactive compounds in the fruits of Tetrapleura tetraptera is widely used in food as a flavouring agent and for spices. In this study, bioactive compounds were extracted by solid-liquid extraction process and the yield was optimized by response surface methodology (RSM) and artificial neural network (ANN). The process parameters optimized were the extraction temperature, particle size and extraction time. Box-Behnken Design was used to study the effect of the process parameters on the extract yield. A quadratic model was obtained by RSM which was used to predict the extract yield. While for ANN, Bayesian Regularization learning algorithm with hyperbolic function (Tanh) for both hidden and output layers was the best model for predicting the extract yield. The performance of both models was established based on their $\mathrm{R}^{2}$ and RMSE values. $\left(\mathrm{R}^{2}\right.$ and RMSE values were 0.9391 and 3.10 for RSM and 0.9637 and 0.8193 for ANN respectively). ANN gave the maximum extract yield of $29.15 \%$ higher than that of RSM which evaluated a yield of $27.70 \%$ with optimum conditions at extraction temperature of $90^{\circ} \mathrm{C}$, particle size of $3.26 \mathrm{~mm}$ and extraction time of 50 mins. It was therefore concluded that ANN is better than RSM in the modeling and optimization of the extraction process parameters.
\end{abstract}

\section{DOI: https://dx.doi.org/10.4314/jasem.v24i2.18}

Copyright: Copyright (C) 2020 Oyedoh et al. This is an open access article distributed under the Creative Commons Attribution License (CCL), which permits unrestricted use, distribution, and reproduction in any medium, provided the original work is properly cited.

Dates: Received: 16 November 2019; Revised: 11 January 2020; Accepted: 22 February 2020

Keywords: Tetrapleura tetraptera, bioactive compounds, process parameters, optimization

In the ancient past, man has been able to harness the remedial effects of plants even before microorganisms and the diseases they cause became known. With this, there has been a growing interest in the discovery and research of these plants. Medicinal plants such as Tetrapleura tetraptera is a deciduous forest plant that belongs to the Mimosaceae family and is mostly found in the West and Central African rainforest zone (Adesina et al., 2016). Commonly called "Aridan/Aidan" in Yoruba, "Imiminje" in Etsako and "Ighimiakia" in Bini, the plant is known for its use in traditional medicine (Adesina et al., 2016). In West Africa, the fruits find their application in drug preparation and production for ailments like fever, arthritis, constipation, inflammation, epilepsy, hypertension, jaundice, post-partum contraction, schistosomiasis, asthma, malaria, microbial infections and pain. The fruits contribute to the diets and wellbeing of indigenous Nigerians in the Southern and Eastern part as it is being used as ingredients for "pepper soup" (Adesina et al., 2016). Researchers have discovered the fruit possess bioactive compounds or phytochemicals, minerals and nutrient contents. These bioactive components which have a physiological action on humans, animals and even micro-organisms include tannins, alkaloids, polyphenols, flavonoids, steroids, saponins, reducing compound, anthraquinones, phlobatannins, glycosides and hydroxynmethyl anthraquinones (Edet et al., 2016). With these bioactive components present, Tetrapleura tetraptera is fit for being used in food and drug production. A bioactive compound is simply defined as a substance having a biological activity on living organism. This biological activity could either be positive or negative depending on the quantity (Azmir et al., 2013). These bioactive compounds from plant materials go through qualitative and quantitative studies that depend mostly on the selection of the right extraction method (Azmir et al., 2013).

In the study of medicinal plant, extraction is the first step which is important in determining the results and outcome of the study. Extraction technique is used in separating the different types of bioactive compounds from plant materials for characterization. The classical techniques used to obtain bioactive compounds from plants include solvent extraction, maceration, and percolation. Solvent extraction is widely being used for extracting variable and desired bioactive compounds from various natural sources (Azmir et al., 
2013). The aqueous and ethanol extract of Tetrapleura tetraptera fruits has been proven to contain the aforementioned bioactive compounds such as 9, 12Octadecenoic acid, 6-Octadecenoic acid and nDecanoic acid. However, these extracts are analyzed using GC-MS (gas chromatography with mass spectrometry) analysis to separate and identify the various bioactive compounds desired. Factors such as temperature, particle size, time and agitation speed determine the extract yield. To maximize extract yield, the extract factors are optimized using statistically designed experiments. In the last two decades, Artificial Neural Networks (ANN) has proven to be much more powerful in modeling and simulation in the various engineering fields, and for predicting the behaviour of non-linear functions. ANN works like the human brain. Hence it is formed by a series of "neurons" (or "nodes") that are organized in layers (Amato et al., 2013; Ding et al., 2013; Zhang, 2014). While comparing optimization using RSM and ANN, M.Mourabet et al., (2014) reported ANN to be a better optimization method.

\section{MATERIALS AND METHODS}

Collection of plant material: Tetrapleura tetraptera fruit was obtained from a local market in Egor Local Government Area, Benin City, Edo state. The fruit was then transported in a polythene bag to the Department of Plant Biology and Biotechnology, University of Benin, Benin City for identification.

Preparation of Tetrapleura tetraptera plant powder and extract: Fresh fruits of the plant were sun-dried for 5 days and chopped into small chips before the extraction process. After chopping the sample into small chips, the sample was further dried under the sun for two weeks so as to reduce the moisture content as low as possible. The dried sample was then ground into different particle sizes and later sieved to obtain 1 $\mathrm{mm}, 3 \mathrm{~mm}$ and $5 \mathrm{~mm}$ as the various particle sizes, before being used as feed for the extraction process. Twenty grams $(20 \mathrm{~g})$ of the dried sample was subjected to extraction with $200 \mathrm{ml}$ of ethanol being used as the solvent by Soxhlet apparatus. The extract obtained for different runs were stored in sample bottles for further analysis.

$$
\% Y E=\frac{\text { mass of crude extract }}{\text { mass of sample }} \times \frac{100}{1} \%
$$

Where $\% \mathrm{YE}=$ percent yield extract

Identification of the components using GC-MS: Computer searches on a NIST Version - Year 2014 were used as mass spectrum data library and the mass spectrum of the unknown compound as compared with the spectrum of known compound. The name of the compound present in the extract yield were identified. Also, the relative percentage of each of the extract constituents was expressed as percentage with peak area.
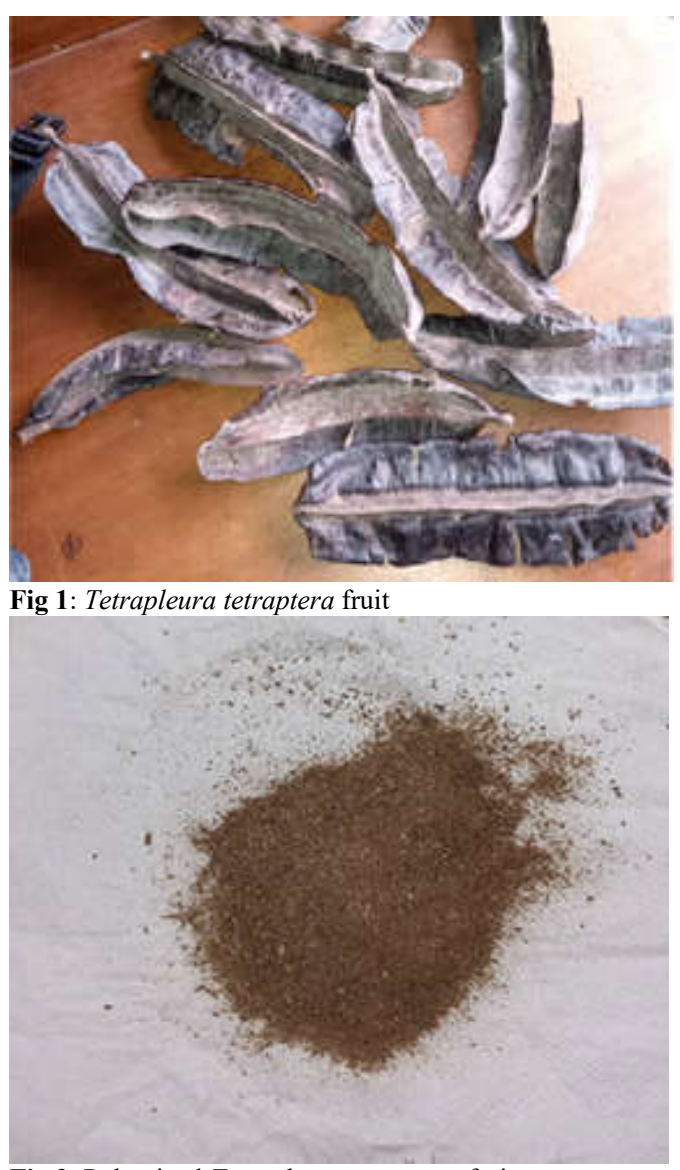

Fig 2: Pulverized Tetrapleura tetraptera fruit

Design of Experiments: A three-factor, three-level Box-Behnken experimental design was employed in order to optimize the extract yield from the Tetrapleura tetraptera sample. The selected extraction process factors for the extract yield were temperature $\left({ }^{\circ} \mathrm{C}\right)$, particle size $(\mathrm{mm})$ and time of extraction $(\mathrm{min})$. The levels of variables optimized are shown in Table 1.

Table 1: Coded and actual levels of the factors for three factor Box-Behnken design

\begin{tabular}{lllll}
\hline Variables & Symbols & \multicolumn{3}{c}{ Coded and Actual levels } \\
\cline { 3 - 5 } & & -1 & 0 & 1 \\
\hline Extraction temperature $\left({ }^{\circ} \mathrm{C}\right)$ & $\mathrm{X}_{1}$ & 78 & 84 & 90 \\
Particle Size (mm) & $\mathrm{X}_{2}$ & 1 & 3 & 5 \\
Extraction time (mins) & $\mathrm{X}_{3}$ & 10 & 30 & 50 \\
\hline
\end{tabular}

The Design Expert $^{\circledR}$ 11.1.2.0 (Stat-ease, Inc. Minneapolis, USA), a statistical software was used to develop the experimental design using Box-Behnken 
Design in Table 1 above to generate 21 experimental runs for the extraction process. The experiments were therefore performed in a random manner in order to maximize the effects of unexplained variability in the observed responses due to extraneous factors.

Response Surface Methodology (RSM): Response surface methodology (RSM) was used to model and optimize the extraction process. The data obtained from the extracted were analyzed statistically so as to fit the quadratic polynomial equation (model) generated by Design Expert $^{\circledR}$ 11.1.2.0 (Stat-ease, Inc. Minneapolis, USA). To correlate the response variable (extract yield) to the independent variables (extraction time, temperature and particle size), multiple regression was used to fit the coefficient of their polynomial model of the response. The fitted polynomial equation is as follows;

$Y_{i}=b_{o}+\sum b_{i} x_{j}+\sum b_{i j} x_{i} x_{j}+\sum b_{i i} x_{i}^{2}+e_{i}(\mathbf{2})$

Where: $Y_{i}$ is the dependent variable or predicted response (extract yield); $b_{o}$ is the offset term/intercept term; $b_{i}(I=1,2,3, \ldots k)$ is the regression coefficient/first order model coefficient ; $b_{i j}$ is the interaction effect; $b_{i i}$ is the quadratic coefficient of $x i$; $\mathrm{x}_{\mathrm{i}}$ and $\mathrm{x}_{\mathrm{j}}$ are independent variables; $\mathrm{e}_{\mathrm{i}}$ is the error term/random error

The Design Expert software thereafter develops the regression model in terms of the actual values that describes the extract yield from the extraction process. The optimal values of the independent variables for the extract yield were obtained by solving eqn. (2.1) and the yield of these optimum conditions predicted by the model was also recorded. The quality of fit of the model was evaluated using test of significance and analysis of variance (ANOVA). Artificial Neural Network Design: A commercial software MATLAB version R2018b (Mathworks Inc., Natick, MA, USA) was used to model the extraction process. The extract yield was predicted using the Neural Network Fitting Tool. This network was trained by different learning algorithms such as Levenberg-Marquardt, Bayesian Regularization and Scaled Conjugate Gradient. The artificial neural network architecture consisted of an input layer with three neurons, an output layer with one neuron and a hidden layer with nine neurons. Each ANN was trained using a default stopping criteria of 1000 iterations. The Box-Behnken design experimental data were divided into three sets which are training, validation and testing data set. The training set is used for computing the gradient and updating the network weights and biases. As the network begins to over-fit the data, the error increases, the training is stopped, and the weights and biases of the epoch, with minimum validation errors are returned as the final ANN structure. In this study, a data set of 15 was used for training, 3 data sets were used for validation while the remaining 3 were used for testing.

Comparison of $A N N \&$ RSM performance: In order to check the accuracy of the models from ANN and RSM, coefficient of determination, $\mathrm{R}^{2}$ and Root Mean Squared Error, RMSE was evaluated using Eqn. 3 and 5 below. While Eqn. 4 gives the Mean Squared Error, MSE used to calculate Eqn. 5;

$$
\begin{aligned}
& R^{2}=1-\sum_{i=1}^{n}\left[\frac{\left(y_{\text {pred }}-y_{\text {exp }}\right)^{2}}{\left(y_{\text {exp }}-y_{\text {avg exp }}\right)^{2}}\right] \\
& M S E=\frac{1}{n} \sum_{i=1}^{n}\left(y_{\text {pred }}-y_{\text {exp }}\right)^{2} \\
& R M S E=M S E^{\frac{1}{2}}
\end{aligned}
$$

Where; $\mathrm{n}=$ number of experimental data; $\mathrm{y}_{\text {pred }}=$ calculated values from model; $\quad y_{\exp }=$ average experimental values; $y_{\text {avg, } \exp }=$ average experimental values

A high value of $\mathrm{R}^{2}$ and a low value of RMSE represent a goodness of fit of the model, which suggest that the model proves suitable for the adequate representation of the actual relationship between the dependent/response variable (extract yield) and the independent variables (extraction time, extraction temperature and particle size).

\section{RESULTS AND DISCUSSION}

Bioactive Components in Tetrapleura tetraptera: In order to identify the bioactive compounds in the fruit and determine their various intensities, GC-MS analysis was carried using ethanol as an extract. GCMS analysis revealed the presence of Dodecanoic acid, Tetradecanoic acid, 9, 12-Octadecadienoic acid (Z, Z), Linoelaidic acid, and others as shown in Fig. 3 and Table 2. From Table 2 and Fig. 3 it was observed that Linoelaidic acid, 9,17-Octadecadienal, (Z) and 9,12-Octadecadienoic acid $(Z, Z)$ had the largest peak area of $20.26 \%$ and retention time of 16.408 minutes which has been reported by Hema R., (2011) to prevent cancer, act as anti-inflammatory, and also help prevent damage to the liver. Erukainure et al. (2017) also reported Tetrapleura tetraptera fruit peels to have bioactive compounds such as 9,12-Octadecenoic acid, 6-Octadecenoic acid and $n$-Decanoic acid which was also observed to be discovered in this research study. 
Table 2: Bioactive compounds in Tetrapleura tetraptera

\begin{tabular}{|c|c|c|c|c|}
\hline $\begin{array}{c}\text { Peak } \\
\text { no. }\end{array}$ & Compounds & $\begin{array}{l}\text { Retention } \\
\text { time }\end{array}$ & Area (\%) & Reported Bioactivity \\
\hline 1 & Dodecanoic acid & 8.926 & 0.33 & Antimicrobial (Anzaku et al., 2017) \\
\hline 2 & Tetradecanoic acid & 11.372 & 0.62 & $\begin{array}{l}\text { Larvicidal and repllent activity (Abubakar and Majinda, } \\
\text { 2016) }\end{array}$ \\
\hline 3 & $\begin{array}{l}\text { Hexadecanoic acid, methyl ester } \\
\text { Pentadecanoic acid, 14-methyl-, methyl } \\
\text { ester }\end{array}$ & 13.118 & 0.11 & $\begin{array}{l}\text { Antibacterial and antifungal (Abubakar and Majinda, } \\
\text { 2016) }\end{array}$ \\
\hline 4 & $\begin{array}{l}\text { n-Hexadecanoic acid } \\
\text { Tridecanoic acid }\end{array}$ & 13.859 & 3.72 & $\begin{array}{l}\text { Anti-inflammatory, Antioxidant, hypocholesterolemic } \\
\text { nematicide, pesticide, anti-androgenic flavour, } \\
\text { hemolytic, 5-Alpha reductase inhibitor, potent mosquito } \\
\text { larvicid (Abubakar and Majinda, 2016) }\end{array}$ \\
\hline 5 & $\begin{array}{l}\text { 9,12-Octadecadienoic acid }(\mathrm{Z}, \mathrm{Z}) \\
\text { Linoelaidic acid }\end{array}$ & 14.408 & 0.18 & $\begin{array}{l}\text { Antiinflammatory, hypocholesterolemic cancer } \\
\text { preventive, hepatoprotective, nematicide, insectifuge, } \\
\text { antihistaminic antieczemic, antiacne, 5-Alpha reductase } \\
\text { inhibitor, antiandrogenic, antiarthritic, anticoronary, } \\
\text { insectifuge (Hema R., 2011) }\end{array}$ \\
\hline 6 & $\begin{array}{l}\text { 11-Octadecenoic acid, methyl ester } \\
\text { 9-Octadecenoic acid, methyl ester }\end{array}$ & 15.490 & 0.35 & $\begin{array}{l}\text { Anti-spasmodic and immune modulators (Gokul and } \\
\text { Priya, 2019). }\end{array}$ \\
\hline 7 & $\begin{array}{l}\text { 9,17-Octadecadienal, }(\mathrm{Z}) \\
\text { Linoelaidic acid } \\
\text { 9,12-Octadecadienoic acid }(\mathrm{Z}, \mathrm{Z})\end{array}$ & 15.848 & 0.28 & $\begin{array}{l}\text { Antiinflammatory, hypocholesterolemic cancer } \\
\text { preventive, hepatoprotective, nematicide, insectifuge, } \\
\text { antihistaminic antieczemic, antiacne, 5-Alpha reductase } \\
\text { inhibitor, antiandrogenic, antiarthritic, anticoronary, } \\
\text { insectifuge (Hema R., 2011) }\end{array}$ \\
\hline 8 & $\begin{array}{l}\text { Linoelaidic acid } \\
\text { 9,17-Octadecadienal, (Z) } \\
\text { 9,12-Octadecadienoic acid (Z,Z) }\end{array}$ & 16.408 & 20.26 & $\begin{array}{l}\text { Antiinflammatory, hypocholesterolemic cancer } \\
\text { preventive, hepatoprotective, nematicide, insectifuge, } \\
\text { antihistaminic antieczemic, antiacne, 5-Alpha reductase } \\
\text { inhibitor, antiandrogenic, antiarthritic, anticoronary, } \\
\text { insectifuge (Hema R., 2011) }\end{array}$ \\
\hline 9 & Octadecanoic acid & 16.692 & 5.85 & $\begin{array}{l}\text { Antioxidant, hypoglycemic and thyroid inhibiting } \\
\text { properties (Ramasamy, 2019) }\end{array}$ \\
\hline 10 & 9,12-Octadecadienoic acid $(\mathrm{Z}, \mathrm{Z})$ & 17.004 & 3.44 & $\begin{array}{l}\text { Antiinflammatory, hypocholesterolemic cancer } \\
\text { preventive, hepatoprotective, nematicide, insectifuge, } \\
\text { antihistaminic antieczemic, antiacne, 5-Alpha reductase } \\
\text { inhibitor, antiandrogenic, antiarthritic, anticoronary, } \\
\text { insectifuge (Hema R., 2011) }\end{array}$ \\
\hline 11 & $\begin{array}{l}\text { Linoelaidic acid } \\
9,12 \text {-Octadecadienoic acid }(\mathrm{Z}, \mathrm{Z})\end{array}$ & 17.348 & 0.72 & $\begin{array}{l}\text { Antiinflammatory, hypocholesterolemic cancer } \\
\text { preventive, hepatoprotective, nematicide, insectifuge, } \\
\text { antihistaminic antieczemic, antiacne, 5-Alpha reductase } \\
\text { inhibitor, antiandrogenic, antiarthritic, anticoronary, } \\
\text { insectifuge (Hema R., 2011) }\end{array}$ \\
\hline 12 & 9,12-Octadecadienoic acid $(\mathrm{Z}, \mathrm{Z})$ & 17.577 & 3.28 & $\begin{array}{l}\text { Antiinflammatory, hypocholesterolemic cancer } \\
\text { preventive, hepatoprotective, nematicide, insectifuge, } \\
\text { antihistaminic antieczemic, antiacne, 5-Alpha reductase } \\
\text { inhibitor, antiandrogenic, antiarthritic, anticoronary, } \\
\text { insectifuge (Hema R., 2011) }\end{array}$ \\
\hline 13 & $\begin{array}{l}\text { cis-9-Hexadecenal } \\
\text { 13-Tetradecenal } \\
\text { Cyclopentadecanone, 2-hydroxy- }\end{array}$ & 18.376 & 1.54 & $\begin{array}{l}\text { Antimicrobial, Anticancer, Hepatoprotective, Anti- } \\
\text { arthritic, anti-asthama, diuretic (Ramasamy, 2019) }\end{array}$ \\
\hline 14 & $\begin{array}{l}9,17 \text {-Octadecadienal, }(\mathrm{Z}) \\
9,12 \text {-Octadecadienoic acid }(\mathrm{Z}, \mathrm{Z}) \\
\text { Linoelaidic acid }\end{array}$ & 20.347 & 0.61 & $\begin{array}{l}\text { Antiinflammatory, hypocholesterolemic cancer } \\
\text { preventive, hepatoprotective, nematicide, insectifuge, } \\
\text { antihistaminic antieczemic, antiacne, 5-Alpha reductase } \\
\text { inhibitor, antiandrogenic, antiarthritic, anticoronary, } \\
\text { insectifuge (Hema R., 2011) }\end{array}$ \\
\hline 15 & $\begin{array}{l}\text { Linoelaidic acid } \\
9,12 \text {-Octadecadienoic acid }(\mathrm{Z}, \mathrm{Z})\end{array}$ & 20.738 & 0.20 & $\begin{array}{l}\text { Antiinflammatory, hypocholesterolemic cancer } \\
\text { preventive, hepatoprotective, nematicide, insectifuge, } \\
\text { antihistaminic antieczemic, antiacne, 5-Alpha reductase } \\
\text { inhibitor, antiandrogenic, antiarthritic, anticoronary, } \\
\text { insectifuge (Hema R., 2011) }\end{array}$ \\
\hline 16 & $\begin{array}{l}\text { 9,12-Octadecadienoyl chloride, }(\mathrm{Z}, \mathrm{Z}) \\
\text { 9,12-Octadecadienoic acid }(\mathrm{Z}, \mathrm{Z}) \\
\text { 9,17-Octadecadienal, }(\mathrm{Z})\end{array}$ & 21.031 & 1.30 & Anticancer (Abubakar and Majinda, 2016) \\
\hline 17 & $\begin{array}{l}\text { 9,12-Octadecadienoic acid }(\mathrm{Z}, \mathrm{Z}) \\
\text { Oleic Acid }\end{array}$ & 21.419 & 0.32 & $\begin{array}{l}\text { Antibacterial (Abubakar and Majinda, 2016) } \\
\text { 5-Alpha-Reductase-Inhibitor, Allergenic, } \\
\text { AlphaReductase-Inhibitor, Anemiagenic, Antialopecic, } \\
\text { Antiandrogenic, Antiinflammatory, Antileukotriene-D4 } \\
\text { (Anti-platelet activating factor), Cancer-Preventive, } \\
\text { Choleretic, Dermatitigenic Flavor, Hypocholesterolemic, } \\
\text { Insectifuge Irritant, Percutaneostimulant, Perfumery, } \\
\text { Propecic (Hema R., 2011) }\end{array}$ \\
\hline 18 & $\begin{array}{l}\text { 9,12-Octadecadienoyl chloride, }(\mathrm{Z}, \mathrm{Z}) \\
9,12 \text {-Octadecadienoic acid }(\mathrm{Z}, \mathrm{Z}) \\
\text { Linoelaidic acid }\end{array}$ & 21.771 & 0.60 & $\begin{array}{l}\text { Antiinflammatory, hypocholesterolemic cancer } \\
\text { preventive, hepatoprotective, nematicide, insectifuge, } \\
\text { antihistaminic antieczemic, antiacne, 5-Alpha reductase } \\
\text { inhibitor, antiandrogenic, antiarthritic, anticoronary, } \\
\text { insectifuge (Hema R., 2011) }\end{array}$ \\
\hline 19 & $\begin{array}{l}\text { 9,17-Octadecadienal, }(\mathrm{Z}) \\
\text { Linoelaidic acid } \\
\text { 9,12-Octadecadienoic acid }(\mathrm{Z}, \mathrm{Z})\end{array}$ & 24.450 & 1.25 & $\begin{array}{l}\text { Antiinflammatory, hypocholesterolemic cancer } \\
\text { preventive, hepatoprotective, nematicide, insectifuge, } \\
\text { antihistaminic antieczemic, antiacne, 5-Alpha reductase }\end{array}$ \\
\hline
\end{tabular}




\begin{tabular}{|c|c|c|c|c|}
\hline & & & & $\begin{array}{l}\text { inhibitor, antiandrogenic, antiarthritic, anticoronary, } \\
\text { insectifuge (Hema R., 2011) }\end{array}$ \\
\hline 20 & $\begin{array}{l}9,12 \text {-Octadecadienoic acid }(\mathrm{Z}, \mathrm{Z}) \\
\text { Linoelaidic acid }\end{array}$ & 24.807 & 0.61 & $\begin{array}{l}\text { Antiinflammatory, hypocholesterolemic cancer } \\
\text { preventive, hepatoprotective, nematicide, insectifuge, } \\
\text { antihistaminic antieczemic, antiacne, 5-Alpha reductase } \\
\text { inhibitor, antiandrogenic, antiarthritic, anticoronary, } \\
\text { insectifuge (Hema R., 2011) }\end{array}$ \\
\hline 21 & $\begin{array}{l}\text { Linoelaidic acid } \\
9,12 \text {-Octadecadienoic acid }(\mathrm{Z}, \mathrm{Z})\end{array}$ & 25.803 & 0.11 & $\begin{array}{l}\text { Antiinflammatory, hypocholesterolemic cancer } \\
\text { preventive, hepatoprotective, nematicide, insectifuge, } \\
\text { antihistaminic antieczemic, antiacne, 5-Alpha reductase } \\
\text { inhibitor, antiandrogenic, antiarthritic, anticoronary, } \\
\text { insectifuge (Hema R., 2011) }\end{array}$ \\
\hline 22 & $\begin{array}{l}\text { 9,12-Octadecadienoyl chloride, }(\mathrm{Z}, \mathrm{Z}) \\
\text { 9,17-Octadecadienal, }(\mathrm{Z}) \\
\text { 9,12-Octadecadienal }\end{array}$ & 26.095 & 0.13 & $\begin{array}{l}\text { Antiinflammatory, hypocholesterolemiccancer } \\
\text { preventive, hepatoprotective, nematicide, insectifuge, } \\
\text { antihistaminic } \\
\text { antieczemic, antiacne, 5-Alpha reductase inhibitor, } \\
\text { antiandrogenic, antiarthritic, anticoronary, insectifuge }\end{array}$ \\
\hline 23 & $\begin{array}{l}\text { 9,17-Octadecadienal, }(\mathrm{Z}) \\
\text { Linoelaidic acid } \\
\text { 9,12-Octadecadien-1-ol, }(\mathrm{Z}, \mathrm{Z})\end{array}$ & 27.078 & 1.03 & $\begin{array}{l}\text { Antiinflammatory, hypocholesterolemic cancer } \\
\text { preventive, hepatoprotective, nematicide, insectifuge, } \\
\text { antihistaminic antieczemic, antiacne, 5-Alpha reductase } \\
\text { inhibitor, antiandrogenic, antiarthritic, anticoronary, } \\
\text { insectifuge (Hema R., 2011) }\end{array}$ \\
\hline 24 & $\begin{array}{l}\text { Linoelaidic acid } \\
\text { 2-Chloroethyl linoleate } \\
\text { 9,17-Octadecadienal, (Z) }\end{array}$ & 27.436 & 0.17 & Flavouring agent (Thomas et al., 2013) \\
\hline 25 & $\begin{array}{l}\text { Dodecanoic acid, 1,2,3-propanetriyl } \\
\text { ester } \\
\text { Dodecanoic acid, 1-(hydroxymethyl)- } \\
\text { 1,2-ethanediyl ester }\end{array}$ & 30.352 & 8.85 & $\begin{array}{l}\text { Antioxidant Antibacterial, COX-1 \& COX-2 inhibitor, } \\
\text { Antiviral, Hypocholesterolemic, Candidicide. }\end{array}$ \\
\hline 26 & $\begin{array}{l}\text { Dodecanoic acid, 1,2,3-propanetriyl } \\
\text { ester } \\
\text { Dodecanoic acid, 1-(hydroxymethyl)- } \\
\text { 1,2-ethanediyl ester }\end{array}$ & 31.146 & 13.95 & $\begin{array}{l}\text { Antioxidant Antibacterial, COX-1 \& COX-2 inhibitor, } \\
\text { Antiviral, Hypocholesterolemic, Candidicide. }\end{array}$ \\
\hline 27 & $\begin{array}{l}\text { Dodecanoic acid, 1,2,3-propanetriyl } \\
\text { ester }\end{array}$ & 31.521 & 10.72 & $\begin{array}{l}\text { Antioxidant Antibacterial, COX-1 \& COX-2 inhibitor, } \\
\text { Antiviral, Hypocholesterolemic, Candidicide. }\end{array}$ \\
\hline 28 & $\begin{array}{l}\text { Dodecanoic acid, 1,2,3-propanetriyl } \\
\text { ester }\end{array}$ & 32.052 & 13.50 & $\begin{array}{l}\text { Antioxidant Antibacterial, COX-1 \& COX-2 inhibitor, } \\
\text { Antiviral, Hypocholesterolemic, Candidicide. }\end{array}$ \\
\hline 29 & $\begin{array}{l}\text { Dodecanoic acid, 1,2,3-propanetriyl } \\
\text { ester } \\
\text { Dodecanoic acid, 1-(hydroxymethyl) - } \\
\text { 1,2-ethanediyl ester }\end{array}$ & 32.218 & 5.98 & $\begin{array}{l}\text { Antioxidant Antibacterial, COX-1 \& COX-2 inhibitor, } \\
\text { Antiviral, Hypocholesterolemic, Candidicide. }\end{array}$ \\
\hline
\end{tabular}

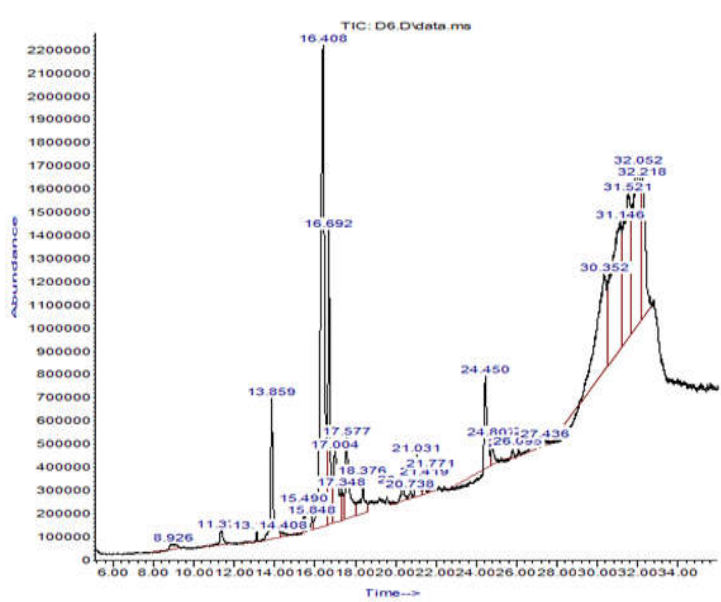

Fig 3: GC-MS chromatogram of Tetrapleura tetraptera ethanolic extract

Response Surface Methodology: Based on the experimental results of BBD in Table 3, a quadratic polynomial was established to identify the relationship between extract yield and process variables. The resulting RSM model equation is following:

Yield $=+514.81971-12.75541 X_{1}+$ $5.89324 X_{2}-0.967139 X_{3}-0.009207 X_{1} X_{2}+$

$$
\begin{gathered}
0.022209 X_{1} X_{3}+0.025278 X_{2} X_{3}+ \\
0.077051 X_{1}^{2}-0.970945 X_{2}^{2}-0.010059 X_{3}^{2}
\end{gathered}
$$

From Eqn. 6, it can be seen that the extraction temperature and extraction time, have negative effect on the extract yield compared to the particle size which has a positive effect on the extract yield when analyzed linearly, while the quadratic effect of the extraction temperature was positive and negative for both particle size and extraction time (M.Mourabet et al., 2014; Rajkovic et al., 2016). With the positive effects of independent variables revealed that their positive changes can cause an increase in the response value (Rajkovic et al., 2016). The experimental results and the predicted values obtained from the model (Eqn. 6) were compared. According to Fig. 4 it was found that the predicted values were close to matching the experimental values with an $\mathrm{R}^{2}$ value of 0.9391 . This implies that $94 \%$ of the variations for extract yield were explained by the independent variables, and this also signifies that the model does not explain about $6 \%$ of variation. Also, the value of the adjusted determination of coefficient $\left(\mathrm{R}_{\mathrm{a}}{ }^{2}=0.8892\right)$ was also high showing a significance of the model. 
Table 3: Three variable Box-Behnken design for the extraction process optimization by using RSM and ANN

\begin{tabular}{|c|c|c|c|c|c|c|c|c|c|}
\hline \multirow{3}{*}{$\begin{array}{l}\text { Run } \\
\text { order }\end{array}$} & \multicolumn{6}{|c|}{ Factors } & \multirow{2}{*}{\multicolumn{3}{|c|}{ Extract Yield (\%) }} \\
\hline & \multicolumn{3}{|c|}{ Coded values } & \multicolumn{3}{|c|}{ Actual values } & & & \\
\hline & $\mathrm{X}_{1}$ & $\mathrm{X}_{2}$ & $\mathrm{X}_{3}$ & $\mathrm{X}_{1}$ & $\mathrm{X}_{2}$ & $\mathrm{X}_{3}$ & $\begin{array}{l}\text { Experimental } \\
\text { (Actual) }\end{array}$ & $\begin{array}{l}\text { RSM } \\
\text { predicted }\end{array}$ & $\begin{array}{l}\text { ANN } \\
\text { predicted }\end{array}$ \\
\hline 1 & 1 & -1 & 0 & 90 & 1 & 30 & 17.80 & 17.70 & 17.49 \\
\hline 2 & 1 & -1 & 1 & 90 & 1 & 50 & 18.51 & 22.74 & 19.72 \\
\hline 3 & -1 & -1 & -1 & 78 & 1 & 10 & 0 & 0 & 1.16 \\
\hline 4 & -1 & -1 & 0 & 78 & 1 & 30 & 8.83 & 7.54 & 8.55 \\
\hline 5 & -1 & -1 & 1 & 78 & 1 & 50 & 7.86 & 7.26 & 8.82 \\
\hline 6 & 0 & -1 & -1 & 84 & 1 & 10 & 0 & 0 & 0.84 \\
\hline 7 & 0 & -1 & 0 & 84 & 1 & 30 & 9.88 & 9.85 & 10.81 \\
\hline 8 & 0 & -1 & 1 & 84 & 1 & 50 & 13.63 & 12.23 & 11.64 \\
\hline 9 & -1 & 0 & -1 & 78 & 3 & 10 & 0 & 2.87 & 0.070 \\
\hline 10 & -1 & 0 & 0 & 78 & 3 & 30 & 8.93 & 11.64 & 7.94 \\
\hline 11 & -1 & 0 & 1 & 78 & 3 & 50 & 11.41 & 12.37 & 11.91 \\
\hline 12 & 0 & 0 & 1 & 84 & 3 & 50 & 17.82 & 17.23 & 18.06 \\
\hline 13 & 1 & 0 & 0 & 90 & 3 & 30 & 23.98 & 21.58 & 23.80 \\
\hline 14 & 1 & 0 & 1 & 90 & 3 & 50 & 31.17 & 27.63 & 29.15 \\
\hline 15 & -1 & 1 & -1 & 78 & 5 & 10 & 0 & 0 & 0.023 \\
\hline 16 & 0 & 1 & -1 & 84 & 5 & 10 & 0 & 0 & 0.10 \\
\hline 17 & 0 & 1 & 0 & 84 & 5 & 30 & 5.05 & 10.06 & 5.49 \\
\hline 18 & 1 & 1 & -1 & 90 & 5 & 10 & 0.44 & 2.57 & 1.43 \\
\hline 19 & 1 & 1 & 0 & 90 & 5 & 30 & 18.96 & 17.69 & 18.83 \\
\hline 20 & 1 & 1 & 1 & 90 & 5 & 50 & 23.8 & 24.75 & 30.84 \\
\hline 21 & -1 & 1 & 0 & 78 & 5 & 30 & 10.59 & 7.97 & 2.26 \\
\hline
\end{tabular}

Table 4 gives the results of the quadratic response surface model fitting in the form of analysis of variance (ANOVA). The analysis of variance is important to check for significance and adequacy of the model. It subdivides the total variation of the results in two sources of variation, the model and the experimental error, shows whether the variation from the model is significant when compared to the variation due to residual error (M.Mourabet et al., 2014). The Fisher's F-test value, which is the ratio between the mean square of the model and the residual error, performs this comparison.

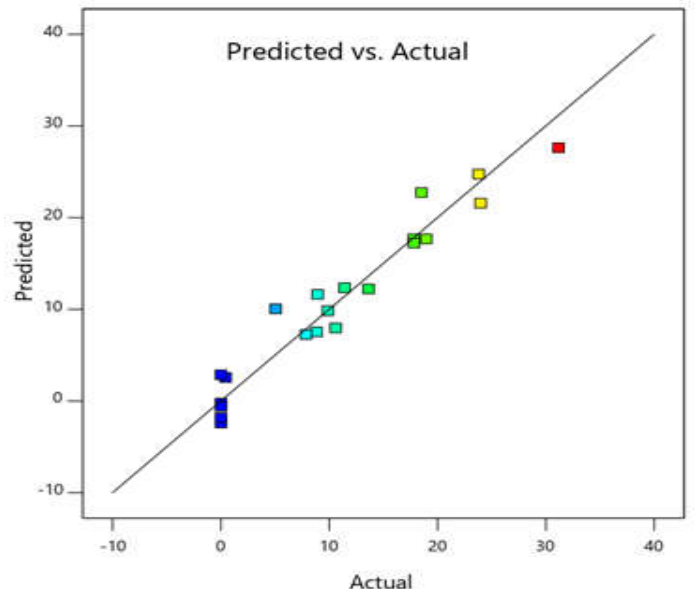

Fig 4: A plot of Predicted against Actual values of the response yield

If the model is a good predictor of the experimental results, F-value should be greater than the tabulated value of F-distribution for a certain number of degrees of freedom in the model at a level of significance (M.Mourabet et al., 2014).
Table 4: Analysis of Variance for RSM - Quadratic Model

\begin{tabular}{|c|c|c|c|c|c|}
\hline Source & $\begin{array}{l}\text { Sum of } \\
\text { Squares }\end{array}$ & dff & $\begin{array}{l}\text { Mean } \\
\text { Square }\end{array}$ & $\begin{array}{l}\text { F } \\
\text { Value }\end{array}$ & Prob $>F$ \\
\hline Model & 1625.23 & 9 & 180.58 & 18.84 & $<0.0001$ \\
\hline $\mathrm{X}_{1}-$ Extraction Temperature & 318.53 & 1 & 318.53 & 33.23 & 0.0001 \\
\hline $\mathrm{X}$-Particle Size & 0.1445 & i & 0.1445 & 0.0151 & 0.9045 \\
\hline $\mathrm{X}$-Extraction Time & 562.09 & i & 562.09 & 58.64 & $<0.0001$ \\
\hline $\mathrm{X}_{1} \mathrm{X}_{2}$ & 0.0842 & 1 & 0.0842 & 0.0088 & 0.9270 \\
\hline $\mathrm{X}_{1} \mathrm{X}_{3}$ & 39.74 & i & 39.74 & 4.15 & 0.0666 \\
\hline $\mathrm{X}_{2} \mathrm{X}_{3}$ & 5.74 & i & 5.74 & 0.5993 & 0.4552 \\
\hline $\mathrm{X}_{1}^{2}$ & 29.24 & i & 29.24 & 3.05 & 0.1085 \\
\hline $\mathrm{X}^{2}$ & 53.04 & 1 & 53.04 & 5.53 & 0.0383 \\
\hline $\mathrm{X}^{2}$ & 63.69 & 1 & 63.69 & 6.64 & 0.0257 \\
\hline Residual & 105.45 & 11 & 9.59 & & \\
\hline Cor Total & 1730.68 & 20 & & & \\
\hline
\end{tabular}

Table 5: Statistical information on Box-Behnken Design

\begin{tabular}{llll}
\hline Std. Dev. & 3.10 & R-Squared & 0.9391 \\
Mean & 10.89 & Adj R-Squared & 0.8892 \\
C.V. \% & 8.40 & Pred R-Squared & 0.7788 \\
PRESS & 382.85 & Adeq Precision & 14.0526 \\
\hline
\end{tabular}

The F-value obtained, 18.84, is greater than the $\mathrm{F}$ value (5.75 at 95\% significance) obtained from the standard distribution table, confirming the adequacy of the model fits. In addition, the p-value was found to be $<$ 0.0001 , which indicated that the model was highly statistically significant. The "Lack of Fit Test" compares the residual error to the pure error from replicated design points. The "Lack of Fit F-value" was not provided by the model. Hence, this model cannot be validated fit. The significance of each term was determined by $p$-value (Prob $>$ F), which is listed in Table 2. As seen in this table that the terms $X_{1}$ and $X_{3}$ were significant $(p<0.05)$ while the other term coefficients were not significant $(p>0.05)$. 
Analysis of Response Surface Plots: 3D response surfaces and the contour plots of the extract yield as a function of extraction temperature, particle size and extraction time are given in Fig. 5 - 7. These plots showed the patterns of the effects of extraction temperature, particle size and extraction time in each individual response.

Effect of extraction temperature and particle size Figure 5 shows the response surface plot and the corresponding contour plot of the combined effect of extraction temperature and particle size on the extract yield. It was observed from the diagram that at low particle size $(1 \mathrm{~mm})$, with an increase in extraction temperature, the extract yield increases. Also, it is observed that at moderate particle size $(3 \mathrm{~mm})$ and high extraction temperature value $\left(89^{\circ} \mathrm{C}\right)$, the extract yield is seen to be at its maximum.
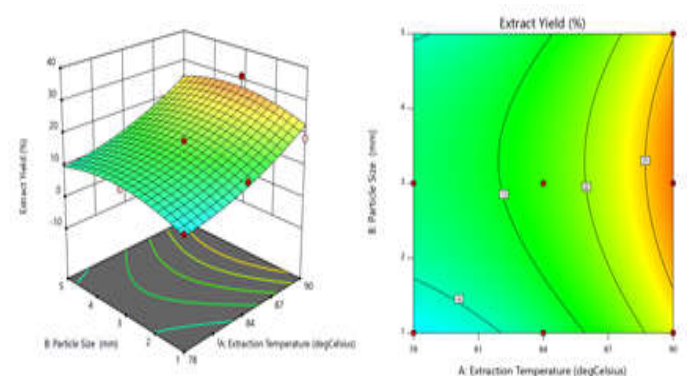

Fig 5: Response surface plot and corresponding contour plot of the simultaneous effect of extraction temperature and particle size

Effect of extraction time and extraction temperature: From Fig. 6, it was observed that at low temperature of about $78{ }^{\circ} \mathrm{C}$, an increase in the extraction time causes an increase in the extract yield. An increase in temperature was discovered to favour the extract yield at any extraction time which shows how much of an influence temperature has on the extraction process. With this, the optimum extract yield proved to be at a high temperature of about $88{ }^{\circ} \mathrm{C}$ to $90{ }^{\circ} \mathrm{C}$ and an extraction time of $43 \mathrm{~min}$ to $50 \mathrm{~min}$.

Effect of extraction time and particle size: From the Fig. 7, it was observed that at low particle size, the extract yield increases slightly with increase in extraction time. Also, at a high extraction time of 49.06 min, a steady increase of the particle size from $1 \mathrm{~mm}$ to $3.21 \mathrm{~mm}$ increases the extract yield with the maximum yield at $27.51 \%$ after which, the yield decreases with a continuous increase of the particle size.
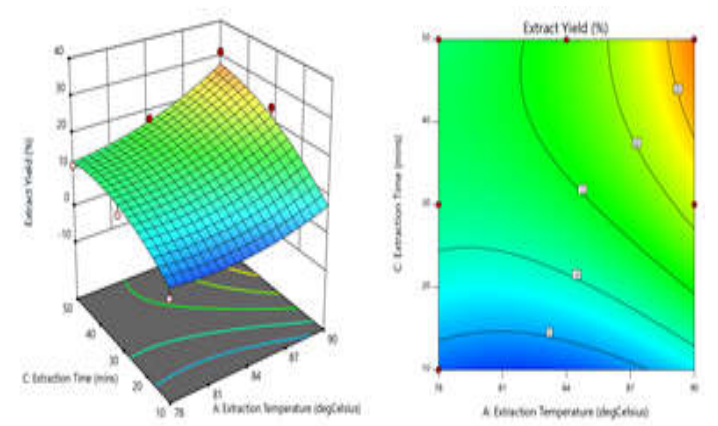

Fig 6: Response surface plot and corresponding contour plot of the simultaneous effect of extraction temperature and extraction time
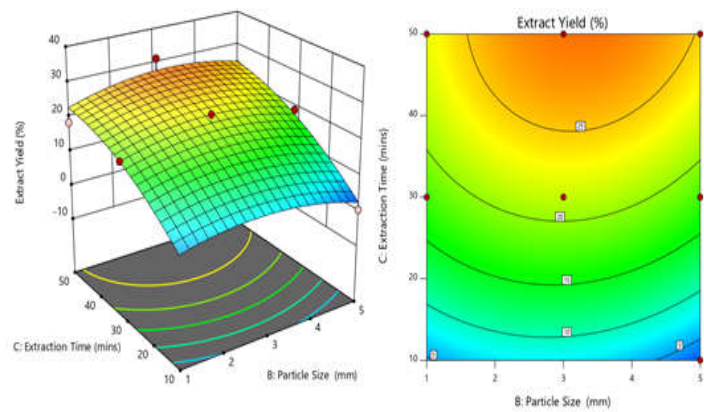

Fig 7: Response surface plot and corresponding contour plot of the simultaneous effect of particle size and extraction time

Optimization using research surface methodology (RSM): To determine the optimal conditions of the independent variables affecting the extract yield obtained from Tetrapleura tetraptera fruit, threedimensional (3D) response surface and contour plots were constructed according to regression model. The optimal condition for this process was established as $\mathrm{X}_{1}=90{ }^{\circ} \mathrm{C} \mathrm{X}_{2}=3.26 \mathrm{~mm} \mathrm{X}_{3}=49.99 \mathrm{~min}$. The predicted extract yield under this optimal condition was $\mathrm{Y}=27.6964 \%$ with a desirability of 0.889 as shown in Fig. 8. It was observed that at a moderate particle size and high extraction temperature had significant effect on the extract yield obtained in this research work.

Artificial Neural Network: A two-layer feed forward network with sigmoid hidden neurons and linear output neurons was used to model the extract. Three training algorithms; Levenberg-Marquadt, Bayesian Regularization and Scaled Conjugate Gradient were used to train the network. Each of these learning algorithms uses $70 \%$ of the experimental data for training the network so as to get used to the experimental data, $15 \%$ of the experimental data is for validating the model and the remaining $15 \%$ is then used for testing the network. 


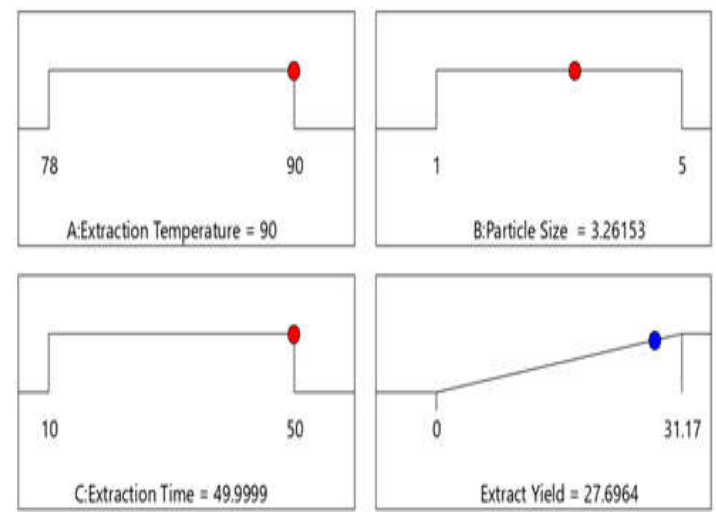

Desirability $=0.889$

Fig 8: Numerical optimization of input variables

Table 6: The different learning algorithms for ANN

\begin{tabular}{lll}
\hline Learning Algorithm & $\mathrm{R}^{2}$ & RMSE \\
Levenberg-Marquadt & 0.82517 & 5.2686 \\
Bayesian-Regularization & $0.9637^{*}$ & $0.81927^{*}$ \\
Scaled Conjugate Gradient & 0.70282 & 10.9044 \\
\hline
\end{tabular}

*preferred

From Table 6, the Bayesian Regularization training algorithm was the best to predict the extract yield. The number of neurons used in the hidden layer were two which resulted in a network topology of (3-9-1); three input factors in the input layer, two neurons in the hidden layer and one output layer. The Bayesian Regularization network was chosen since it gave the least RMSE and the highest $\mathrm{R}^{2}$ value of 0.81927 and 0.9637 respectively. The architecture of the ANN model is shown in the figure 10 .

Comparison of RSM and ANN Performance: To determine the best model that accurately optimize the effect of extraction temperature, particle size and extraction time on the extract yield, the $\mathrm{R}^{2}$ and the RMSE values of both models evaluated and their results are shown in Table 7 . The result showed that both models used for optimization produced accurate prediction due to their high $\mathrm{R}^{2}$ values. However, ANN gave a lower RMSE value when compared to the RSM model. Therefore, ANN was a better modeling tool due to its low RMSE and high extract yield.

Conclusion: The bioactive compounds identified in Tetrapleura tetraptera will be useful in the manufacture of drugs to combat certain ailments and beneficial for the production of food spices. RSM and ANN were used to model and determine the optimum process parameters that give the high extract yield. With the ANN model providing a higher yield and $\mathrm{R}^{2}$ value than RSM, it is suggested that the ANN model be used to optimize the process of bioactive compounds extraction from Tetrapleura tetraptera fruit.
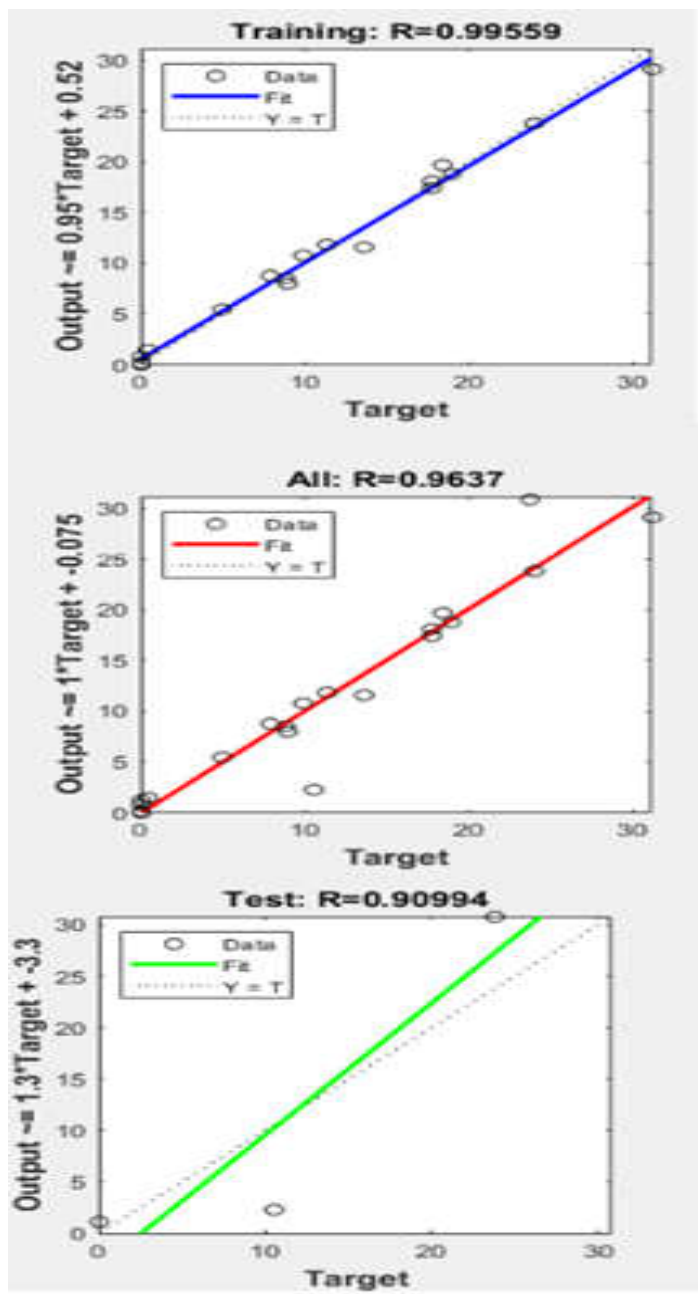

Fig 9: Bayesian Regularization training algorithm

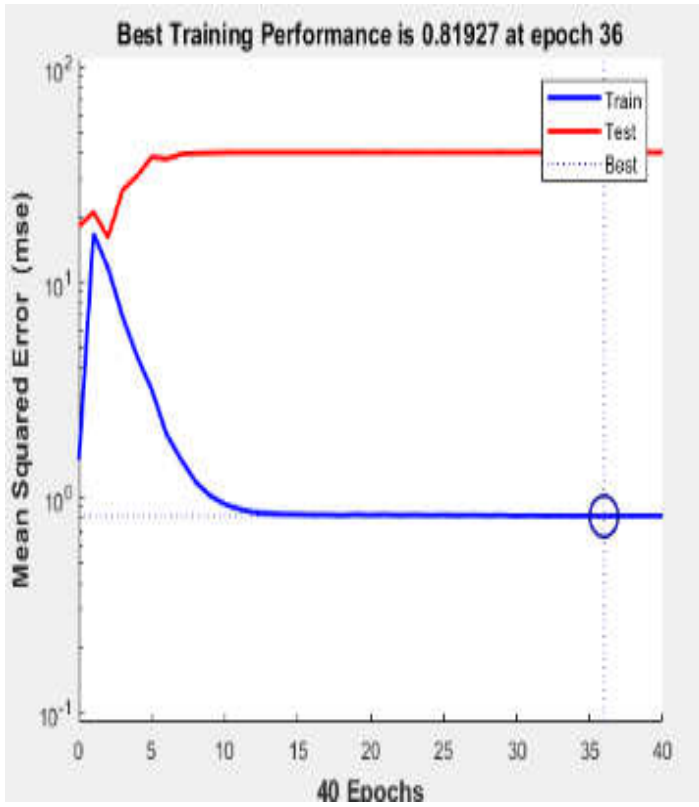

Fig 10: MSE of the Bayesian Regularization algorithm 


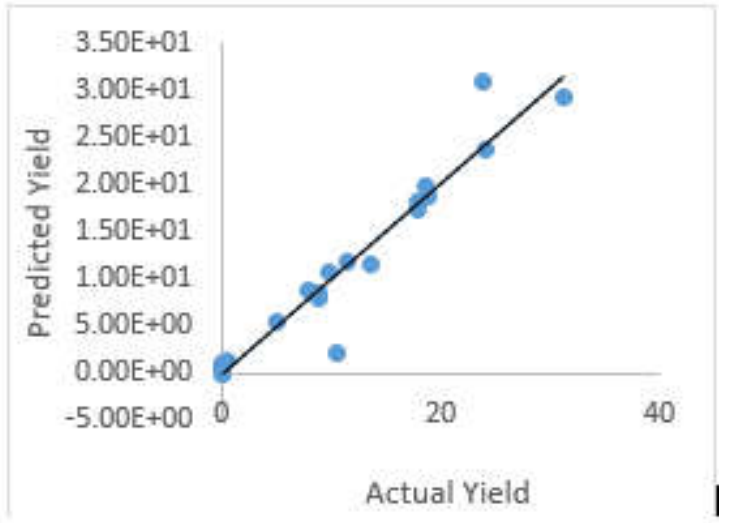

Fig 11: A plot of Predicted vs Actual values by ANN

Table 7: Comparison of RSM and ANN

\begin{tabular}{lllllll}
\hline $\begin{array}{l}\text { Model Extraction } \\
\text { temperature size } \\
\text { ("C) }\end{array}$ & $\begin{array}{l}\text { Particle Extraction } \\
\text { (mme }\end{array}$ & $\begin{array}{l}\text { Extract } R^{2} \\
\text { (mins) }\end{array}$ & $\begin{array}{l}\text { RMSeld } \\
\text { (\%) }\end{array}$ & \\
\hline RSM & 90 & 3.26 & 50 & 27.696 & 0.9391 & 3.10 \\
ANN & 90 & 3 & 50 & 29.15 & 0.9637 & 0.81927 \\
\hline
\end{tabular}

\section{REFERENCES}

Abubakar, M; Majinda, R (2016). GC-MS Analysis and Preliminary Antimicrobial Activity of Albizia adianthifolia (Schumach) and Pterocarpus angolensis (DC). Medicines, 3(1), 3

Adesina, SK; Iwalewa, EO; Johnny, II (2016). Tetrapleura tetraptera Taub - Ethnopharmacology , Chemistry, Medicinal and Nutritional Values- A Review. 12(3), 1-22

Amato, F; López, A; Peña-méndez, EM; Vaňhara, P; Hampl, A (2013). Artificial neural networks in medical diagnosis. pp. 47-58.

Anzaku, AA; Akyala, JI; Juliet, A; Obianuju, EC (2017). Antibacterial Activity of Lauric Acid on Some Selected Clinical Isolates. Annals of Clinical and Laboratory Research, 05(02), 1-5.

Azmir, J; Zaidul, ISM; Rahman, MM; Sharif, KM; Mohamed, A; Sahena, F; Jahurul, MHA; Ghafoor, K; Norulaini, NAN; Omar, AKM (2013). Techniques for extraction of bioactive compounds from plant materials: A review. Journal of Food Engineering. Elsevier Ltd, 117(4), pp. 426-436

Ding, S; Li, H; Su C; Yu, J; Jin F (2013). Evolutionary artificial neural networks : a review. pp. 251-260.

Edet, UO; Ebana, RUB; Ekanemesang, UM; Ikon, GM; Etok, CA; Edet, AP (2016). Antimicrobial
Activity, Phytochemical Screening and Nutrient Analysis of Antimicrobial Activity, Phytochemical Screening and Nutrient Analysis of Tetrapleura tetraptera and Piper guineense. (February 2018). doi: 10.9734/AJMAH/2016/29362.

Erukainure, OL; Onifade, OF; Odjobo, BO; Olasehinde, TA; Adesioye, TA; Tugbobo-Amisu, AO; Adenekan, SO; Okonrokwo, GI (2017). Ethanol extract of Tetrapleura tetraptera fruit peels : Chemical characterization, and antioxidant potentials against free radicals and lipid peroxidation in hepatic tissues. Integrative Medicine Research. Taibah University, 11(6), 861-867

Gokul, K; Priya, V (2019) Phytochemical and GC-MS analysis of $\mathrm{Z}$ iziphus glabrata Heyne ex Roth ( Rhamnaceae ). 6(2), 42-51.

Hema, R; Kumaravel, S; Alagusundaram, K (2011). GC/MS Determination of Bioactive Components of Murraya koenigii. 7(1), 2009-2012.

Mourabet, M; El Rhilassi, A; Bennani-Ziatni, M; Taitai, A (2014). Comparative Study of Artificial Neural Network and Response Surface Methodology for Modelling and Optimization the Adsorption Capacity of Fluoride onto Apatitic Tricalcium Phosphate. Universal Journal of Applied Mathematics, 2(2), 84-91.

Rajkovic, KM; Stojicevic, SS; Velickovic, DT; Nikolic, NC; Simic, VM; Karabegovic, IT; Lazic, LM (2016). Optimization of microwave-assisted extraction of total polyphenolic compounds from chokeberries by response surface methodology and artificial neural network. 160, 89-97

Ramasamy, M; Santhiya, N (2019). Parreysia corrugata ( Muller 1774 ) and their pharmacological activities. 9(8), 155-158.

Thomas, E; Annesh TP; Thomas DG; Anandan R (2013). GC-MS analysis of phytochemical compounds present in the rhizomes of Nervilia aragoana GAUD. Asian Journal of Pharmaceutical and Clinical Research, 6(3), 68-74.

Zhang, PG (2014). Neural Networks For Data Mining. (June), doi: 10.1007/978-0-387-09823-4. 